\title{
Packings of frictionless spherocylinders
}

\author{
Claus Heussinger ${ }^{1}$ \\ ${ }^{1}$ Institute for theoretical physics, Georg August University Göttingen, \\ Friedrich Hund Platz 1, 37077 Göttingen, Germany
}

\begin{abstract}
We present simulation results on the properties of packings of frictionless spherocylindrical particles. Starting from a random distribution of particles in space, a packing is produced by minimizing the potential energy of inter-particle contacts until a force-equilibrated state is reached. For different particle aspect ratios $\alpha=10 \ldots 40$, we calculate contacts $z$, pressure as well as bulk and shear modulus. Most important is the fraction $f_{0}$ of spherocylinders with contacts at both ends, as it governs the jamming threshold $z_{c}(\alpha)=8+2 f_{0}(\alpha)$. These results highlight the important role of the axial "sliding" degree of freedom of a spherocylinder, which is a zero-energy mode but only if no end-contacts are present.
\end{abstract}

\section{INTRODUCTION}

Packing and flow of granular particles has been the subject of intense research over many years. Even though granular particles generally have irregular, non-spherical shapes, work has mainly been concerned with the properties of systems of spherical particles. A few studies deal with flow properties of non-spherical particles of different shapes, e.g. cube-like shapes [1, 2], needles [3 5] or simulations with polygons [6] or ellipsoids [7, 8]. Coupling of shear flow to rotational degrees of freedom leads to non-trivial alignment properties [9 11, even in the limit of nearly spherical particles [12, 13].

The jamming properties of nearly spherical, ellipsoidal or spherocylindrical particles have been discussed in some detail. Slightly non-spherical particles can use space more efficiently and pack at optimized, higher densities [14, 15. Frictional interaction forces, however, seem to act against this packing optimization [1]. Jamming of nearly-spherical particles is complicated by the special role of the rotational degrees of freedom 16 19. In the sphere limit rotational degrees of freedom are lost. However, already for nearly-spherical ellipsoids the jamming transition is modified as rotational and translational degrees of freedom form separate bands that only weakly $\operatorname{mix}$ [16.

Here we are concerned with packings of frictionless spherocylindrical particles. We are primarily interested in long, high-aspect-ratio $(\alpha)$ particles and the question how the limiting behavior to $\alpha \rightarrow \infty$ looks like. Infinitely long spherocylinders have a symmetry related to translations along their axis ("sliding"), which cannot be constrained by any interactions. The question is in how far this symmetry is still visible in the jamming of nearly-infinite-length, i.e. finite-length spherocylinders, just as rotational symmetry is still visible in nearly-spherical particles.

Previous work on high-aspect ratio particles has dealt with the jamming of elastic fibers [20, 21], or rods [14, 2224. Frictional interactions have been seen to strongly affect the response to shear deformations [25], possibly leading to a gravity-induced yielding transition as a function of particle length [26, 27].
We will discuss the jamming properties of static packings of soft spherocylinders, as well as their response to small bulk and shear deformations. We will see that the crossover to infinite length is governed by the fraction of particles which have their axial translation constrained by contacts at the spherocylinder ends. This fraction vanishes when $\alpha \rightarrow \infty$.

\section{MODEL}

We study three-dimensional (3d) packings of spherocylindrical particles. Each spherocylinder (SC) $i=$ $1 \ldots N$ consists of a cylindrical part of length $\ell_{i}$ and two hemispherical caps of diameter $d_{i}$ at the two ends. The center-line of the cylinder is called the backbone. The volume of a $\mathrm{SC}$ is thus

$$
V_{\mathrm{sc}}=(\pi / 6) d^{3}+(\pi / 4) d^{2} \ell .
$$

The particles interact via repulsive contact forces similar to those from models for spheres. A contact between particles $i$ and $j$ is established whenever the shortest distance between the backbones, $r_{i j}=\left|\mathbf{r}_{i j}\right|$, is less than their average diameter $d_{i j}=\left(d_{i}+d_{j}\right) / 2$. The distance vector can be written as

$$
\mathbf{r}_{i j}=\mathbf{R}_{i}+\hat{\mathbf{n}}_{i} s_{i}-\left(\mathbf{R}_{j}+\hat{\mathbf{n}}_{j} s_{j}\right),
$$

where $\mathbf{R}_{i}$ is the position of the center of mass of particle $i, \hat{\mathbf{n}}_{i}$ represents the direction of the particle backbone, and $s_{i} \in\left[-\ell_{i} / 2, \ell_{i} / 2\right]$ is the arclength parameter along the backbone that specifies where the shortest distance between $i$ and $j$ is reached. By definition the vector $\mathbf{r}_{i j}$ is perpendicular to both backbones, except for cases where the shortest distance is reached at an end of one or both of the SC (i.e. $s_{i}= \pm \ell_{i} / 2$ ). The actual force is applied halfway along the vector $\mathbf{r}_{i j}$, at the position $\mathbf{y}_{i j}=\hat{\mathbf{n}}_{i} s_{i}+\mathbf{r}_{i j} / 2$ away from the center of mass (with a small correction for unequal-sized particles). This is, in general, very close to the surface of the two particles. The procedure is similar to Ref. 22].

The force $\mathbf{f}_{i j}$ on particle $i$ from the contact with $j$ is directed normally to the particle surface. It is calculated 
as in the Cundall-Strack model 28

$$
\mathbf{f}_{i j}=\left[-k_{n} \delta_{i j}-c_{n} v_{i j}^{n}\right] \hat{\mathbf{n}}_{i j},
$$

Here, the normal direction $\hat{\mathbf{n}}_{i j}=\mathbf{r}_{i j} / r_{i j}$ points from particle $j$ to $i$ at the point of application of the force. The overlap $\delta_{i j}=d_{i j}-r_{i j}$ is a positive quantity.

The velocity $v_{i j}^{n}$ represents the projection of the relative velocity $\mathbf{v}_{i j}^{\text {con }}$ at the contact. The latter derives from the center of mass translational $\mathbf{v}_{i}$ and rotational motion $\boldsymbol{\omega}_{i}$ as $\mathbf{v}_{i j}^{\text {con }}=\mathbf{v}_{i}-\mathbf{v}_{j}-\mathbf{y}_{i j} \times \boldsymbol{\omega}_{i}+\mathbf{y}_{j i} \times \boldsymbol{\omega}_{j}$. The parameter $k_{n}$ is a spring constant, $c_{n}$ a viscous damping constant.

The equations of motion for particle $i$ are

$$
\begin{gathered}
m \ddot{\mathbf{r}}_{i}=\sum_{j} \mathbf{f}_{i j} \\
\mathbf{I}_{i} \cdot \dot{\boldsymbol{\omega}}_{i}=\sum_{j} \mathbf{y}_{i j} \times \mathbf{f}_{i j}
\end{gathered}
$$

where $\mathbf{I}_{i}$ is the moment of inertia of particle $i$ calculated for a spherocylinder with a homogeneous mass density.

The equations of motion are combined with the FIRE algorithm [29] to minimize potential energy. In this algorithm, velocities are rescaled after each time-step to guide the descent in the potential energy landscape.

We have set $k_{n}=1$ and $c_{n}=0$. All particles have the same mass $m=1$ and aspect ratio $\alpha=\ell / d$. Half of the particles have $d=1$, the other half have $d=1.4$. System sizes are chosen such that the linear dimension of the simulation box is at least three times the length of the simulated SC. In terms of particle number this means, $N=3072 \ldots 6144$. The unit of energy is thus $k_{n} d^{2}$, times are expressed in units of the elastic collision time $\sqrt{m / k_{n}}$.

\section{A. Numerical implementation}

We integrate the equations of motion on a GPU using a velocity Verlet algorithm for the translational degrees of freedom, and a Richardson-like iteration for the rotational degrees of freedom (see appendix), which are represented as quaternions. Normalization of the quaternions is ensured by rescaling at each time step.

The dynamics is stopped, when the potential energy does not change appreciably $\left(\Delta E<10^{-6}\right)$ and the kinetic energy is below a threshold $\left(E_{k i n} / N<10^{-12}\right)$. Most of the time the residual kinetic energy is many orders of magnitude smaller than this threshold.

In exceptional cases it may happen that the shortest distance between two nearly parallel spherocylinders jumps discontinuously from one end to the other. This may lead to oscillations which make it impossible to drain the kinetic energy from the system. For nearly parallel $\mathrm{SC}$ we thus include a modification of the distance calculation as follow. The shortest distance betweeen parallel
SCs are taken at the center of the overlap region. For nearly parallel SCs (angle $\theta<\theta_{\text {thres }}$ ) this location is linearly interpolated to the actual shortest distance. As a result no discontinuity arises, and we find immediate relaxation of kinetic energy of previously oscillating systems.

\section{RESULTS}

The pressure tensor is calculated from the virial expression

$$
P_{\alpha \beta}=\frac{1}{V} \sum_{k<l} f_{k l}^{\alpha} R_{k l}^{\beta}
$$

where $\mathrm{V}$ is the volume of the system, $f_{k l}^{\alpha}$ is $\alpha$-component of the force applied on particle $\mathrm{k}$ by particle $\mathrm{l}$ and $R_{k l}^{\beta}$ is the $\beta$-component of the distance between the particles' center of mass. Using the center-of mass coordinates in the pressure tensor is not immediately obvious. Such a definition ensures that the $P_{\alpha \beta}$ is the force in $\alpha$ direction experienced by a hypothetical wall (with normal along $\beta$ direction) that itself consists of spherocylindrical particles. In a previous publication on short SC [11] we have used the $r_{k l}^{\beta}$ instead. For short particles the difference between both definitions is very small.

For the isotropic pressure $p=\operatorname{tr} P / 3=\frac{1}{3 V} \sum_{k<l} \mathbf{f}_{k l}$. $\mathbf{R}_{k l}$ the difference between both definitions vanishes for side-contacts, as $\mathbf{f}_{k l} \perp \hat{\mathbf{n}}_{k}, \hat{\mathbf{n}}_{l}$ and thus

$$
\mathbf{f}_{k l} \cdot \mathbf{r}_{k l}=\mathbf{f}_{k l} \cdot \mathbf{R}_{k l} \quad \text { (side contacts), }
$$

see Eq. (2). Below we will find that side-contacts are dominant whenever SCs are long enough.

\section{A. Jammed configurations}

With the procedure just described a set of jammed packings is generated, starting from a spatially random initial distribution. Effects from ordering can be

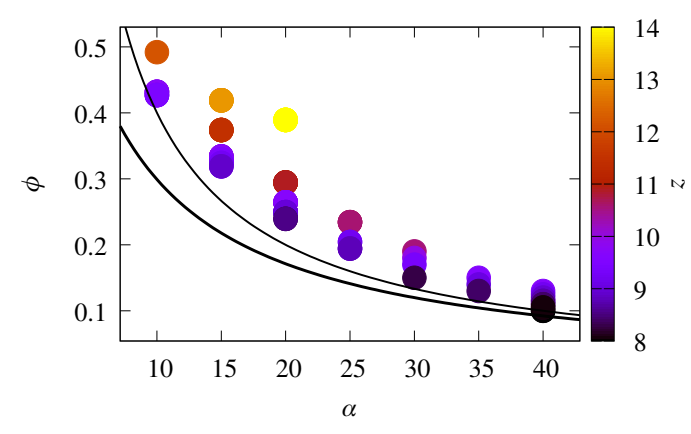

FIG. 1. State-points in the plane spanned by volume-fraction $\phi$ and aspect-ratio $\alpha$. Color code given by number of contacts per particle $z$ of the packing. Thin line is $\phi=4 / \alpha$, thick line $\phi=\left(1+\frac{\alpha^{2} / 4}{\alpha+2 / 3}\right)^{-1}$ following from Eq. 10 . 


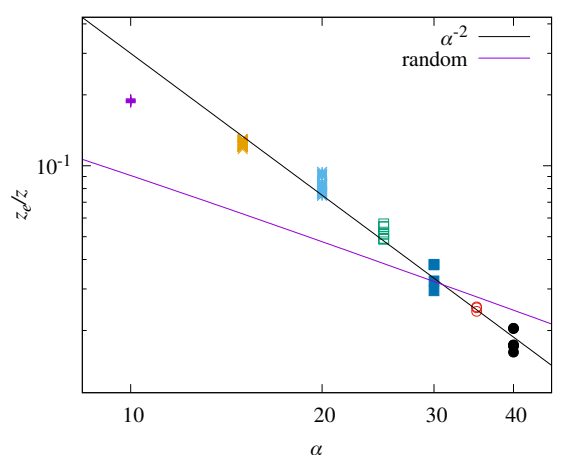

FIG. 2. Ratio of end-to-total contacts $z_{e} / z$ vs. aspect ratio $\alpha$. random: ratio of side-to-total area.

neglected as particles are orientationally constrained and don't move much during minimization. The aspect ratio is varied from $\alpha=10 \ldots 40$ and the associated volume fractions are chosen to approach the jamming threshold. Several packings at the same state point serve to estimate fluctuations.

Fig. 1] shows the state-points of the generated packings in the $\alpha-\phi$ plane and highlights the broad range of $\phi$-values necessary to cover the jamming transition for all aspect ratios. In previous work, Philipse 30 argued that, asymptotically $(\alpha \rightarrow \infty)$, the jamming density is inversly proportional to the aspect ratio, $\phi_{J}=c / \alpha$. The proportionality factor $c$ is given by $c \approx z / 2$, where $z$ is the connectivity, the average number of contacts per particle. Thus, longer SCs do not make more contacts than shorter SCs, but pack at lower density.

In the general case, the Philipse argument relates the number of contacts $z$ of a SC to the number density $\rho=\phi / V_{\text {sc }}$ and the orientationally averaged excluded volume 30, 31

$$
V_{\mathrm{excl}}=(\pi / 2) \ell^{2} d+2 \pi d^{2} \ell+(4 / 3) \pi d^{3}
$$

as

$$
z=\rho V_{\mathrm{excl}}=\phi \frac{V_{\mathrm{excl}}}{V_{\mathrm{sc}}} .
$$

This reduces to the above relation $z=2 \phi \alpha$ in the limit of long SCs, where the end caps are irrelevant. Both, the general expression Eq. 10 and the asymptotic version are drawn in Fig. 1 for $z=8$.

At jamming the number of contacts are constrained by mechanical equilibrium. Maxwell counting 32 for SC particles with three translational and two rotational degrees of freedom (no rotation around the long axis) gives $z_{J}=10$ contacts per particle that are minimally necessary to ensure mechanical equilibrium.

In fact, the axial translational degree of freedom needs special consideration in long SCs. It can only be constrained by contacts at the SC ends, but not by contacts at the sides. As the total number of contacts does not increase with $\ell$, the relative importance of the end contacts is expected to vanish. Fig. 2 shows how the fraction of end contacts $z_{e} / z$ decreases with aspect ratio (roughly as $\alpha^{-2}$ ) in our different packings. Interestingly, if contacts were distributed randomly over the entire surface (or volume) of a SC, the number of end contacts would show a different behavior, $z_{e} \sim \alpha^{-1}$, which is also indicated in the figure. One can thus conclude that asymptotically, the axial translation mode is not constrained, thus Maxwell counting gives $z_{J}=8$, which is the value used in Fig. 1 .

To assess the usefulness of this limit to our packings, we calculate the pressure and relate it to the corresponding connectivity, see Fig. 3 a. At jamming, $z=z_{J}$, the pressure should vanish. Obviously, the data is a mess and neither prefers $z_{J}=8$ nor $z_{J}=10$, but rather an aspect-ratio dependent $z_{c}(\alpha)$. In the following we use $z_{c}$ (and not $z_{J}$ ) whenever the $\alpha$-dependent threshold $z_{c}(\alpha)$ is meant.

For the longest SCs a value $z<8$, i.e. even below Maxwell counting, is observed. This also happens for spherical particles and is a sign of the occurence of rattling particles 33 . Thus, a more refined analysis is warranted. In the general case, a certain number of particles, $N_{\text {ec }}$ has end contacts (on both ends) 34. Accounting in addition for rattling particles $N_{\mathrm{r}}$, i.e. those that do not have any contact (roughly $1 \ldots 5 \%$ ), Maxwell counting gives

$$
z_{c}=8+\frac{2 N_{\mathrm{ec}}}{N-N_{\mathrm{r}}}=8+2 f
$$

with $f$ the fraction of SCs with end contacts. This fraction is plotted in Fig. 35. As expected, longer SCs have lower $f$. Apparently, for each aspect ratio $\alpha$ there is a finite limit $f_{0}(\alpha)$ when approaching the jamming transition, $f_{0} \equiv f\left(z \rightarrow z_{c}\right)$. This is plotted in the inset of the figure. The solid line indicates a dependence $f_{0} \propto \alpha^{-2}$, similar to the fraction of end-contacts in Fig. 2.

In Fig. 3b pressure is plotted again, now against $\delta z \equiv$ $z-z_{c}(f)$. The data appears well ordered and even follows power-laws. The longest SCs $\alpha=40$ seem to suggest direct proportionality between pressure and contacts, giving $p \sim\left(z-z_{c}\right)$. However, it seems that the exponent of the power-law is continuously shifting with aspect ratio. This is rather unusual. Instead, a cross-over from one power-law to another is to be expected. This behavior is because pressure is a combination of different factors the crucial one being (see Eq. (7)) the normalized sum over contacts $1 / V \sum_{\text {contacts }}(\ldots)$, which can be written as $(\phi z / \ell)\langle\ldots\rangle_{c}$. The observable in brackets is related to the overlaps in the contacts. Thus, pressure variations reflect different contributions from changing overlaps, but also from $z$ and $\phi$, which change appreciably in our ensemble of configurations.

In order to access the properties of the overlaps themselves, one may define a rescaled pressure $\hat{p}=p \ell / z \phi$. Alternatively, one can study the potential energy (per contact) $E / N_{c}=\frac{k}{2}\left\langle\delta^{2}\right\rangle$. Its variations also directly reflects the overlap via the second moment $\left\langle\delta^{2}\right\rangle$ of the distribution of overlap values. In Fig. $4 \mathrm{~A}$ the mean-squared 

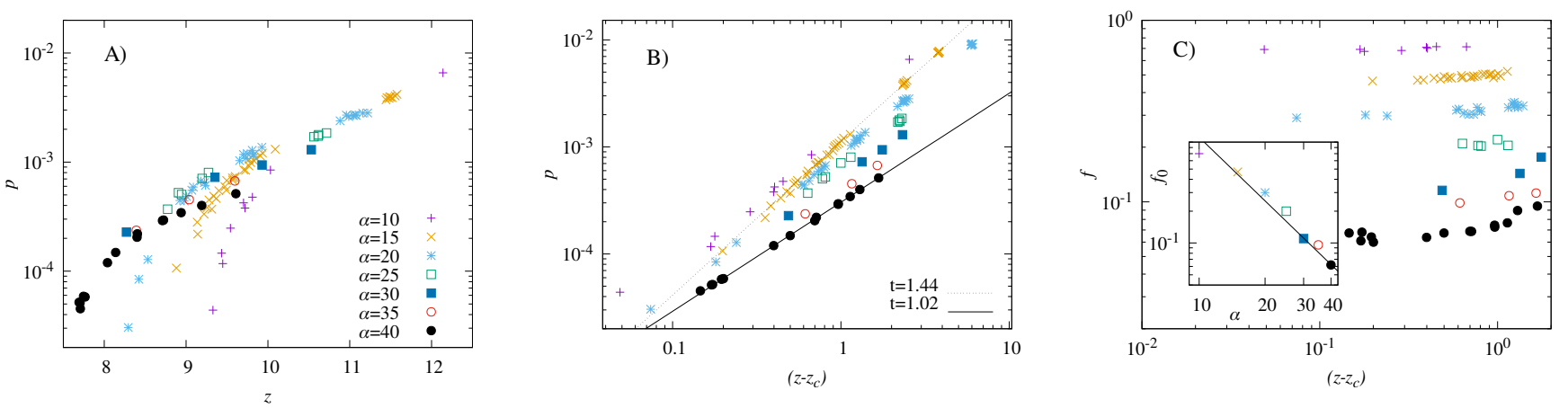

FIG. 3. A) Pressure $p$ vs. connectivity $z$ for different aspect ratios $\alpha=10 \ldots 40$ (rattlers are not accounted for). B) Pressure $p$ vs. reduced connectivity $z-z_{c}$ as defined in Eq. [11. The lines are fits of the form $p \sim\left(z-z_{c}\right)^{t}$ to the $\alpha=15$ and 40 data, respectively. C) Fraction of particles with end contacts, $f$, vs. reduced connectivity $z-z_{c}$ for different aspect ratios $\alpha=10 \ldots 40$. Inset: Limiting value, $f_{0}=f\left(z \rightarrow z_{0}\right)$ vs. aspect ratio $\alpha$. Line is $f_{0} \sim \alpha^{-2}$.

overlap as derived from the potential energy is plotted. The line $\propto\left(z-z_{c}\right)$ points to a regime where $\left\langle\delta^{2}\right\rangle \propto \delta z$
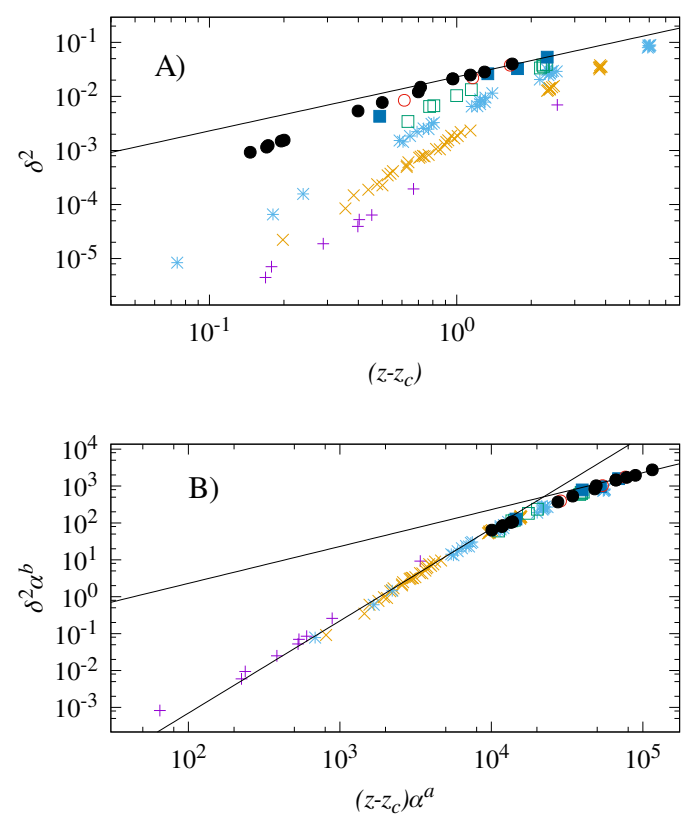

FIG. 4. A) Mean square overlap $\left\langle\delta^{2}\right\rangle$ vs. reduced connectivity $\left(z-z_{c}\right)$ for various $\alpha$; color code as in Fig. 3 . Line is $\delta z^{1}$. B) Axes rescaled by powers of $\alpha$; exponents are determined as $a=b=3$. Lines are $\delta z^{1}$ and $\delta z^{2.5}$.

and independent of SC length. This regime sets in above a connectivity scale $\delta z^{\star} \sim \alpha^{-3}$, as the scaling analysis in panel B shows. There, we plot the scaling ansatz $\left\langle\delta^{2}\right\rangle \sim \alpha^{-b} F_{\delta}\left(\delta z \alpha^{a}\right)$, with a scaling function $F_{\delta}$ and exponents $a=b=3$. As mentioned above, also a rescaled pressure may be used to study the overlaps. In order to access the first moment $\langle\delta\rangle$ a special pressure $p_{s}$ needs to be defined that is calculated from the side contacts only

$$
p_{s}=\frac{1}{3 V} \sum_{\text {side c. }} \mathbf{R}_{c} \cdot \mathbf{f}_{c} \sim \frac{z_{s} \phi}{\ell}\langle\delta\rangle,
$$

where we have used Eq. (8), which is only valid for side contacts. We have checked that corresponding scaling properties emerge from the rescaled pressure $\hat{p}_{s} \equiv$ $p_{s} \ell / z_{s} \phi \sim\langle\delta\rangle \sim \sqrt{\left\langle\delta^{2}\right\rangle}$ as from the potential energy. The effective exponents $t$ seen in Fig. $3 \mathrm{~B}$ can then be understood from the dependence of the overlap $\langle\delta\rangle$ together with the variation of the prefactor $z=z_{c}+\delta z$.

\section{B. Linear response to deformation}

\section{Bulk modulus}

To probe the response to compressive deformations, a quasistatic compression protocol is followed. Starting with a minimized packing, the volume of the simulation box is changed, followed by another minimization. This is repeated several times to be able to record a pressurestrain relation. The strain increment $\Delta \gamma$ is defined from the change of the volume as $\Delta \gamma=-\Delta V / V$, or in other words, $d V / d \gamma=-V$. The modulus $K$ is defined from the slope of the pressure-strain relation, $p(\gamma)=K \gamma$, which is identical to the usual definition of the inverse compressibility $1 / \kappa=-V d p / d V=-V(d p / d \gamma)(d \gamma / d V)=$ $K$. The strain values are chosen small enough such that $p(\gamma)$ is a linear function. It turns out that using $\Delta \gamma=O\left(10^{-5}\right)$ is small enough to obtain $5 \ldots 10$ points over which the function is indeed linear.

Fig. 5A plots the bulk modulus $K$ vs. the reduced connectivity. For large $z-z_{c}$ the modulus increases linearly in $z-z_{c}$. For small $z-z_{c}$ the modulus reaches an $\alpha$ dependent constant value $K_{0}$. This plateau decreases strongly with particle length, roughly as $K_{0} \sim \alpha^{-3}$. At least part of this $\alpha$-dependence stems from the normalization of pressure with volume, Eq. (7). As has been derived in Eq. (12), the $1 / V \sum_{\text {contacts }}$ turns into $\phi z / V_{\mathrm{sc}} \rightarrow z^{2} / \alpha^{2}$. However, the dependence of the bulk modulus is stronger than this factor $\alpha^{-2}$.

In analogy to Eq. (12) a reduced bulk modulus of the side contacts is defined as $\hat{K}_{s}=K_{s} \ell / \phi z_{s}$, where $K_{s}$ is 

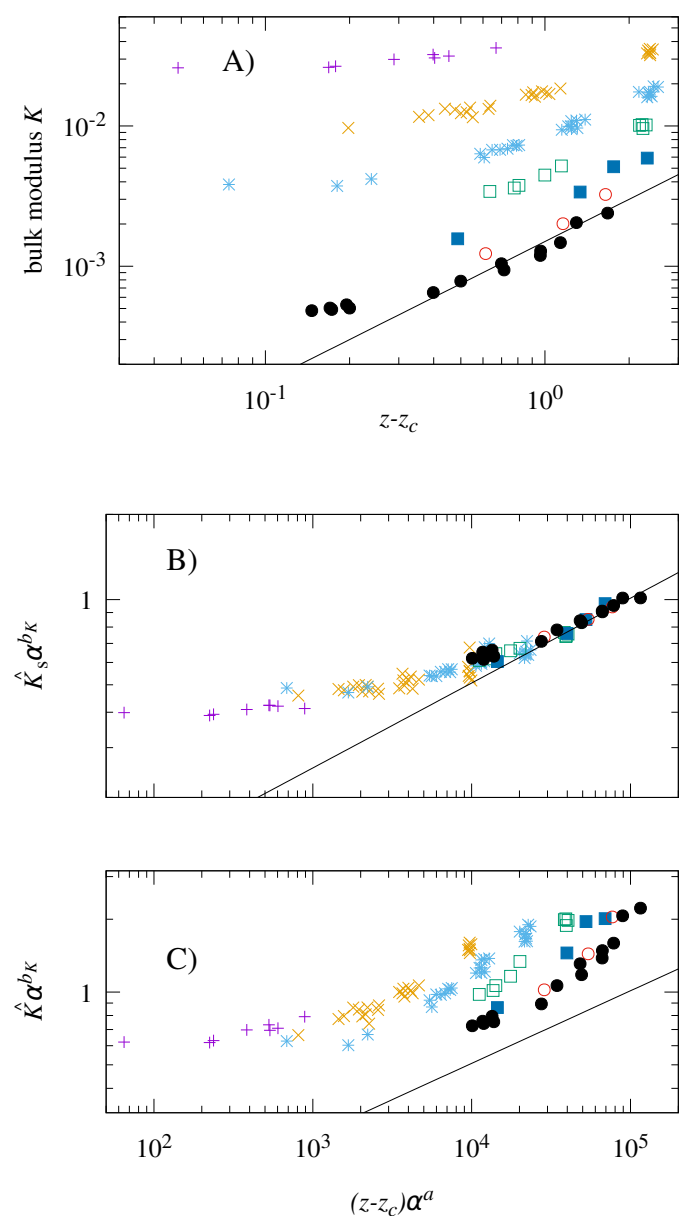

FIG. 5. A) Linear bulk modulus $K$ vs. reduced connectivity $z-z_{c}$. Line is $K=0.0015\left(z-z_{c}\right)$. Color code as in Fig. 6 Aspect ratios $\alpha=10 \ldots 40$ from top to bottom. B) Reduced bulk modulus $\hat{K}_{s}=K_{s} \ell / \phi z_{s}$ vs. $z-z_{c}$ and rescaled by powers of $\alpha, a=3, b_{K}=0.9$. $K_{s}$ is the contribution to the bulk modulus from the side contacts. Line has slope of 0.3 , see text for details. C) Reduced (full) bulk modulus $\hat{K}=K \ell / \phi z$ vs. $z-z_{c}$ and rescaled by powers of $\alpha$. The same exponents are used as in panel B), $a=3, b_{K}=0.9$, but no collapse is achieved. Line as in panel B).

the contribution to the bulk modulus from the side contacts, only. From the definition of the bulk modulus it is clear that it directly represents the overlaps and their changes under compressive deformations. This reduced modulus is plotted in Fig. $5 \mathrm{~B}$, where also the axes are scaled by powers of $\alpha$. Collapse is achieved with a scaling function $F_{K}$ and $\hat{K}_{s}=\alpha^{-b_{K}} F_{K}\left(\delta z \alpha^{a}\right)$, where $a=3$ as for Fig. 4 and $b_{K}=0.9$. The latter value is the missing factor that yields $K_{0} \sim \alpha^{-2.9}$ as also observed in the full bulk modulus. Notably, the full bulk modulus cannot be scaled in this way (see Fig. $5 \mathrm{C}$ ). Beyond the plateau the data suggest a dependence $\widehat{K}_{s} \sim \delta z^{0.3}$. This would also imply that asymptotically the $\alpha$-dependence drops out, $\hat{K}_{s}=\alpha^{-0.9} F_{K}\left(\delta z \alpha^{3}\right) \rightarrow \alpha^{-0.9}\left(\delta z \alpha^{3}\right)^{0.3}=\delta z^{0.3}$.

\section{Shear modulus}

Analogously to the bulk deformations, steps of small shear strains $\delta \gamma=10^{-5}$ are applied via Lees-Edwards boundary conditions [35]. The shear stress $\sigma=P_{x y}$ from Eq. (7) is monitored and its dependence on $\gamma$ fit to a linear function. The stress-strain relation is usually nearly linear, such that a fit yields the linear elastic shear modulus $\mu=d \sigma / d \gamma$.
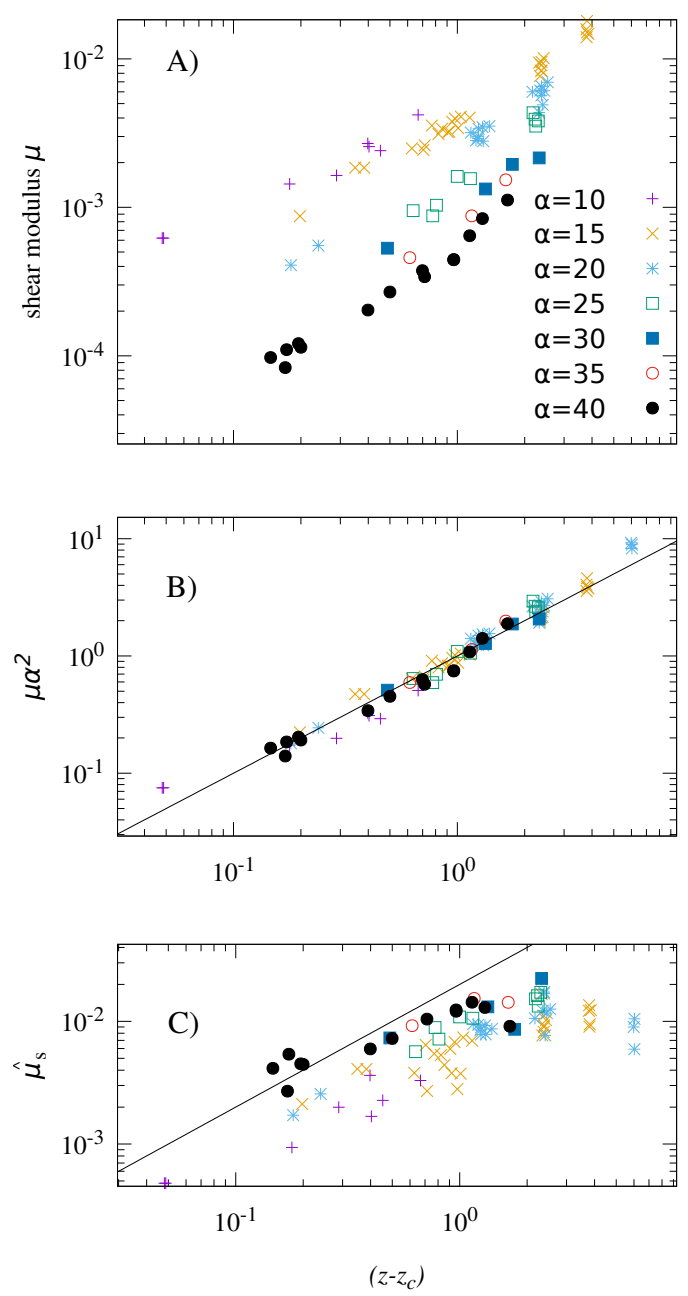

FIG. 6. A) Linear shear modulus $\mu$ vs. reduced connectivity $z-z_{c}$. B) Scaled shear modulus $\mu \alpha^{2}$; line is $\mu \alpha^{2}=\left(z-z_{c}\right)$. C) Reduced shear modulus $\hat{\mu}_{s}=\mu_{s} \ell / \phi z_{s}$ vs. $z-z_{c} \mu_{s}$ is the contribution to the shear modulus from the side contacts. Line has slope of 1 .

In contrast to the bulk modulus, the shear modulus does not present a plateau at small $z-z_{c}$, such that close to jamming $\mu \ll K$. As in the standard scenario for spherical particles $(\alpha=0)$ [36] we find $\mu / K \sim \delta z$. Panel B) of the same figure illustrates in more detail the scaling properties of the shear modulus. By rescaling the y-axis with $\alpha^{2}$ we can show that $\mu=\alpha^{-2}\left(z-z_{c}\right)$. For 
completeness we also display the reduced shear modulus $\hat{\mu}_{s}=\mu_{s} \ell / \phi z_{s}$ of the side contacts $z_{s}$. Surprisingly, the data is rather noisy, much more so than the full modulus. For example, the data for $\alpha=15$ (yellow crosses) show quite some scatter at intermediate $z$, which is much smaller in the full modulus. Apparently, the splitting into the two components from end- and side-contacts is quite variable. A large contribution from the side contacts may be buffered by a small contribution from the end contacts, and vice-versa.

\section{DISCUSSION}

We have presented simulation results on the properties of packings of frictionless spherocylindrical particles.

A packing represents a force-balanced, minimal energy state, given that the spherocylinders (SC) interact via pairwise contact forces at the point of closest approach.

Spherocylinders have a special shape that gives rise to interesting properties. First, rotational symmetry around the axis sets the jamming threshold at $z_{J}=10$ contacts per SC (two contacts per degree of freedom). Second, there is an approximate translational symmetry along the long axis. For contacts at the SC-side interaction forces are directed perpendicular to the long axis. Therefore translation along this direction ("sliding") does not change any of these forces and no resistance builds up. If this symmetry would be perfect, jamming would happen at $z_{J}=8$. Contacts at the SC-end, of course, break the symmetry as they do feel sliding, and resist such motion.

In our simulations we find that packings of long SC have very few end contacts, much less than, e.g. a random distribution of contacts on the surface (or over the volume) of a $\mathrm{SC}$ would suggest. The fraction $f_{0}$ of particles with ends constrained decreases with $\mathrm{SC}$-aspect ratio as $f_{0} \propto \alpha^{-2}$ (see Fig. $3 \mathrm{C}$ ). We can explain this scaling via an extension of Philipse's argument [30] for the jamming density $\phi_{c} \sim z / \alpha$. If SCs are distributed randomly in space, then $f_{0}$ is just the probability that two out of $N$ SCs interesect a given test particle at its two ends. If we define $\pi_{1 e}$ as the probability that the test particle is intersected by one SC at either end, then $f_{0} \approx \frac{N(N-1)}{2} \pi_{1 e}^{2}$. The probability $\pi_{1 e} \approx V_{\mathrm{sc}} / V_{\mathrm{box}} \ll 1$ as the the center of mass of the intersecting SC has to lie within a volume $V_{\mathrm{sc}}$ close to the end of the test particle. As a result, we find $f_{0} \sim \rho^{2} V_{\mathrm{sc}}^{2} \sim \phi_{c}^{2} \sim \alpha^{-2}$ as observed (to be compared to the probability of single contacts at side or end $\pi_{1}=V_{\text {excl }} / V_{\text {box }}$ and $z=N \pi_{1} \sim \phi \alpha$, which is Eq. (10). Given, that only a fraction of particles have their ends constrained, the jamming threshold smoothly interpolates from 10 to 8 , when $f_{0}$ decreases from 1 to 0 . In fact, one can use constraint counting to derive $z_{c}\left(f_{0}\right)=8+2 f_{0}$.

These findings suggest a comparison with the system of ellipsoidal particles [16, 17] that has been mentioned in the introduction. When going from spheres to slightly as- pherical ellipsoids new rotational degrees of freedom are introduced and constraint counting gives a new jamming threshold $z_{J}=12$ (in the general case of three distinct axes). In fact, this threshold is not immediately reached as the new degrees of freedom are either zero-energy (quartic) modes or form a separate rotational band. This only weakly interferes with the jamming threshold. Only at larger asphericity, when rotational and translational degrees of freedom mix, do the zero modes vanish and full ellipsoidal jamming is reached.

In the case of spherocylinders it is the lowering of the $\mathrm{SC}$ length (from infinity) that introduces a new degree of freedom, that of translation along the $\mathrm{SC}$ axis. As long as the ends of the $\mathrm{SC}$ are not constrained, this mode is a zero-energy (but not quartic) mode. For the remaining fraction $f_{0}$ of SCs with ends constrained this translational mode is expected to be of finite-energy and corresponds to the rotational band in the case of ellipsoids. It would be interesting to compute the density of states to see if these modes also form a separate band and if so when (at what SC length) mixing is observed.

The suitable control parameter to measure the distance to jamming then is $\delta z \equiv z-z_{c}\left(f_{0}\right)$ with $f_{0}(\alpha) \sim \alpha^{-2}$. This defintion allows to easily compare different aspect ratios $\alpha$ that generally jam at wildly different volume fractions $\phi_{J}(\alpha) \sim \alpha^{-1}$. Given the control parameter $z-$ $z_{c}$ we present measurements of pressure $p$ and potential energy (or mean-squared overlap $\left\langle\delta^{2}\right\rangle$ ) of the packings, as well as their response to bulk and shear deformation.

The analysis of the parameter dependence is complicated by the simultaneous variation of several quantities. As an example consider Eq. 12, which is an expression for the part of the pressure that stems from the side contacts $z_{s}, p_{s} \sim \frac{z_{s} \phi}{\alpha}\langle\delta\rangle$. In the immediate vicinity to jamming, one can safely set $z_{s}=z_{s J}$ and $\phi=\phi_{J}$ and neglect their variation with the control parameter $\delta z$. The pressure then only changes due to changes of the overlap $\langle\delta\rangle$ with $\delta z$.

As it turns out, we cannot produce packings close enough to jamming to guarantee this limiting behavior. Equilibration times quickly reach time-scales that are no longer practical with our simulation methods. It might be the very sliding motion of end-unconstrained SCs that spoils equilibration, as this motion cannot be resisted. This dilemma is visible when comparing the pressure data in Fig. $3 \mathrm{~B}$ with the averaged overlaps $\left\langle\delta^{2}\right\rangle$ in Fig. 4. The overlaps are characterized by a cross-over scale $\delta z^{\star} \sim \alpha^{-a}$ with $a \approx 3$. On the other hand, such a scale is not visible in the pressure data, which rather feature power-laws with continuously shifting exponents $t \approx 1 \ldots 1.5$. By analyzing $\hat{p}_{s}=\alpha p_{s} / z_{s} \phi$, we have verified that the scale is indeed hidden due to variations of the factors $z$ and $\phi$.

The physical origin of the crossover scale is currently unclear. A clue to its understanding may lie in the rather large numerical prefactor $\delta z^{\star} \approx 2 \cdot 10^{4} \alpha^{-a}$, which is rather unusual. A possible resolution may be to write $\delta z^{\star}$ in terms of $f_{0}$ rather than $\alpha$. With the numeri- 
cal factor taken from Fig. $3 \mathrm{C}$ (inset), we would obtain $\delta z^{\star} \approx 20 f_{0}^{3 / 2}$. A negative exponent $-a=-3$ indicates that asymptotically, i.e. for $\alpha \rightarrow \infty$, the regime $\delta z>\delta z^{\star}$ prevails. For the overlaps, for example, this means $\left\langle\delta^{2}\right\rangle \sim \delta z$ independent of $\alpha$. This scaling means, that each of the $N \delta z$ constraints contributes independently and roughly equally to the potential energy.

A similar scale is observed in the bulk modulus $\hat{K}_{s}$ of the side contacts (see Fig. $5 \mathrm{~B}$ ) which, via its definition $K_{s}=d p_{s} / d \gamma$, represents the change of overlaps with dilational strain, $\hat{K}_{s} \sim d \delta / d \gamma$. In the asymptotic regime the $\alpha$-dependence drops out and $\hat{K}_{s}=\alpha^{-0.9} F_{K}\left(\delta z \alpha^{3}\right) \rightarrow$ $\alpha^{-0.9}\left(\delta z \alpha^{3}\right)^{0.3}=\delta z^{0.3}$

Interestingly, the full bulk modulus $K$, which also includes contacts from the SC ends, shows different properties. It is linear in $\delta z$ and cannot be scaled with $\delta z^{\star}$. It seems reasonable to suppose that this difference is a consequence of the special role of the SC length $\ell$ in the end contacts. For end contacts $\ell$ enters the pressure in a different way than for side contacts. This is apparent in the definition of pressure (Eqs. (7D) via the virial contribution of a contact, $\mathbf{f}_{k l} \cdot \mathbf{R}_{k l}$, where $\mathbf{R}_{k l}$ is the vector between the center-of-masses of the two contacting SCs $k$ and $l$, and thus $\left|\mathbf{R}_{k l}\right| \sim \ell$. Another way of seeing the special $\ell$-dependence in the end-contacts is by considering an affine deformation of the packing. From the properties of an affine map, the distance between the two ends of a SC (being $\ell$ apart) should change by $\propto \gamma \ell$. As the SC itself does not change length, this would naturally induce additional overlaps of exactly this size. On the other hand, side contacts will only experience overlaps $\propto \gamma d$, with the diameter $d$ of the SC.

Closer towards jamming the bulk modulus has a plateau which strongly decreases with SC length. This decrease is stronger than the prefactor $\frac{z \phi}{\alpha}$ might suggest. Thus, the overlaps themselves, or rather their change with strain decreases with SC length, $\delta^{\prime} \sim \alpha^{-0.9}$.

Finally, we also calculate the shear modulus $\mu$ (see Fig. 6). In contrast to the bulk modulus the shear modulus does not have a plateau, but vanishes continuously at jamming, $\mu \sim \alpha^{-2}\left(z-z_{c}\right)$. For the ratio of both we find $\mu / K \sim \delta z \rightarrow 0$, similar to packings with spherical particles 36. The scaling with $\alpha^{-2}$ is again due to the prefactor $z \phi / \alpha$. The splitting of the shear modulus in contributions from side and end contacts does not seem to be reasonable, as the scatter is unexpectedly high. In fact, the distinction between side and end contacts is more important for the pressure as for the shear stress, as Eq. (8) shows.

A vanishingly small shear modulus has also been observed in packings of elastic fibers [25]. As it turns out, by introducing frictional interactions between the fibers, the shear modulus strongly increases 25]. As friction primarily inhibits sliding motion, this supports our understanding that it is the axial sliding degrees of freedom of the SCs that are responsible for the increased shear modulus.

A similar effect is observed in bonded networks of fibers, where permanent bonds take the role of the frictional interactions, and bond/fiber deformation that of overlaps 37, 38. Again, the length-scale $\ell=\alpha d$ plays a special role. If fibers try to slide in response to shear, the anchoring points of fiber bonds will be displaced by an amount $\propto \gamma \ell$. This then is the amount of strain induced locally either in the bonds or the fibers. Note, that this strain is much larger than $\propto \gamma d$ with $d$ a microscopic length-scale like the length of a bond or the distance between bonds.

Future work should analyze these analogies between the different systems in more detail, in particular paying attention to the respective role of the "mesoscopic" length-scale $\ell$. This length is on the one hand much larger than the typical microscopic length-scales as diameter, bond length or mesh-size of the structure, but also supposedly much smaller than the scale of the entire system, be it a granular heap, a fiber network or a polymer mesh.

\section{ACKNOWLEDGMENTS}

Financial support by the German Science Foundation (DFG) via the Heisenberg program (HE-6322/2) is acknowledged.

\section{Appendix A: Integration of rotational degrees of freedom}

The integration of the particle orientation utilizes the equation 39.

$$
\dot{\mathbf{q}}=\frac{1}{2} \mathbf{W}^{\mathrm{T}}(\mathbf{q}) \boldsymbol{\omega}_{4}
$$

with angular velocities $\boldsymbol{\omega}_{4}=(\boldsymbol{\omega}, 0)$ taken in the particle frame.

The updated quaternion $\mathbf{q}_{\text {new }}$ is obtained in a two step process, via

$$
\begin{aligned}
\mathbf{q}_{1} & =\mathbf{q}_{\mathrm{old}}+\left.d t \dot{\mathbf{q}}\right|_{\text {old }} \\
\mathbf{q}_{1 / 2} & =\mathbf{q}_{\mathrm{old}}+\left.(d t / 2) \dot{\mathbf{q}}\right|_{\text {old }} \\
\mathbf{q}_{2} & =\mathbf{q}_{1 / 2}+\left.(d t / 2) \dot{\mathbf{q}}\right|_{1 / 2} \\
\mathbf{q}_{\text {new }} & =2 \mathbf{q}_{2}-\mathbf{q}_{1} .
\end{aligned}
$$

The value of $\left.\dot{\mathbf{q}}\right|_{1 / 2}$ is obtained from $\boldsymbol{\omega}_{1 / 2}$ via the angular momentum $\mathbf{l}$ in the lab frame

$$
\mathbf{l}_{1 / 2}=\mathbf{l}_{\text {old }}+(d t / 2) \mathbf{t}
$$

with the torque $\mathbf{t}$. Transforming to the particle frame

$$
\mathbf{I} \boldsymbol{\omega}_{1 / 2}=\mathbf{R}\left(\mathbf{q}_{\mathbf{1} / \mathbf{2}}\right) \mathbf{l}_{1 / 2}
$$

where $\mathbf{I}$ is the (diagonal) moment of inertia in the particle frame and $\mathbf{R}$ is the rotation matrix transforming from lab to particle frame. 
[1] J. R. Royer, G. L. Burton, D. L. Blair, and S. D. Hudson, Soft Matter 11, 5656 (2015)

[2] C. D. Cwalina, K. J. Harrison, and N. J. Wagner, Soft Matter 12, 4654 (2016)

[3] F. Tapia, S. Shaikh, J. E. Butler, O. Pouliquen, and E. Guazzelli, J. Fluid Mech. 827, R5 (2017).

[4] R. E. Egres and N. J. Wagner, J. Rheol. 49, 719 (2005)

[5] E. Brown, H. Zhang, N. A. Forman, B. W. Maynor, D. E. Betts, J. M. DeSimone, and H. M. Jaeger, Phys. Rev. E 84, 031408 (2011)

[6] F. Alonso-Marroquin and H. J. Herrmann, Phys. Rev. E 66, $021301(2002)$

[7] C. S. Campbell, Phys. Fluids 23, 013306 (2011).

[8] M. Trulsson, J. Fluid Mech. 849, 718740 (2018)

[9] T. Börzsönyi, B. Szabó, G. Törös, S. Wegner, J. Török, E. Somfai, T. Bien, and R. Stannarius, Phys. Rev. Lett. 108, $228302(2012)$

[10] T. Marschall and S. Teitel, Phys. Rev. E 97, 012905 (2018)

[11] T. Nath and C. Heussinger, Eur. Phys. J. E 42, 157 (2019).

[12] T. A. Marschall and S. Teitel, Phys. Rev. E 100, 032906 (2019)

[13] D. B. Nagy, P. Claudin, T. Börzsönyi, and E. Somfai, Phys. Rev. E 96, 062903 (2017)

[14] S. R. Williams and A. P. Philipse, Phys. Rev. E 67, 51301 (2003).

[15] A. Donev, R. Connelly, F. H. Stillinger, and S. Torquato, Phys. Rev. E 75, 051304 (2007)

[16] Z. Zeravcic, N. Xu, A. J. Liu, S. R. Nagel, and W. van Saarloos, Eur. Phys. Lett. 87, 26001 (2009)

[17] M. Mailman, C. F. Schreck, C. S. O'Hern, and B. Chakraborty, Phys. Rev. Lett. 102, 255501 (2009).

[18] K. VanderWerf, W. Jin, M. D. Shattuck, and C. S. O'Hern, Phys. Rev. E 97, 012909 (2018).

[19] M. van Hecke, Journal of Physics: Condensed Matter 22, $033101(2010)$

[20] D. Rodney, M. Fivel, and R. Dendievel, Phys. Rev. Lett. 95, $108004(2005)$
[21] R. S. Hoy, Phys. Rev. Lett. 118, 068002 (2017)

[22] L. Pournin, M. Weber, M. Tsukahara, J.-A. Ferrez, M. Ramaioli, and T. M. Liebling, Granular Matter 7, $119(2005)$.

[23] C. R. A. A. F. W.Tavares and M. Castier, Powder Techn. 134, 167 (2003).

[24] M. Bargieł, in Computational Science - ICCS 2008, edited by M. Bubak, G. D. van Albada, J. Dongarra, and P. M. A. Sloot (Springer Berlin Heidelberg, Berlin, Heidelberg, 2008) pp. 126-135.

[25] C. Barbier, R. Dendievel, and D. Rodney, Phys. Rev. E 80, 016115 (2009)

[26] M. Trepanier and S. V. Franklin, Phys. Rev. E 82, 011308 (2010).

[27] H. Tapia-McClung and R. Zenit, Phys. Rev. E 85, 061304 (2012)

[28] P. A. Cundall and O. D. L. Strack, Géotechn. 29, 47 (1979).

[29] E. Bitzek, P. Koskinen, F. Gähler, M. Moseler, and P. Gumbsch, Phys. Rev. Lett. 97, 170201 (2006).

[30] A. P. Philipse, Langmuir 12, 1127 (1996).

[31] L. Onsager, Ann. N. Y. Acad. Sci. 51, 627 (1949).

[32] C. R. Calladine, Int. J. Solids Struct. 14, 161 (1978).

[33] C. Heussinger and J.-L. Barrat, Phys. Rev. Lett. 102, 218303 (2009)

[34] Spherocylinders with contacts at only one end do hardly ever occur in equilibrated states. During minimization the SC can easily (without resistance) slide in the opposite direction to remove these contacts.

[35] A. W. Lees and S. F. Edwards, J. Phys. C: Solid State Phys. 5, 1921 (1972)

[36] C. S. O'Hern, L. E. Silbert, A. J. Liu, and S. R. Nagel, Phys. Rev. E 68, 011306 (2003).

[37] J. Plagge, A. Fischer, and C. Heussinger, Phys. Rev. E 93, $062502(2016)$

[38 C. Heussinger, B. Schaefer, and E. Frey, Phys. Rev. E 76, 031906 (2007)

[39] D. C. Rapaport, The Art of Molecular Dynamics Simulation, 2nd ed. (Cambridge University Press, 2004). 